\title{
The Robust Passive Location Algorithm for Maneuvering Target Tracking
}

\author{
Xiaojun Yang, Gang Liu, Jinku Guo, Hongqiao Wang, and Bing He
}

Xian Research Institute of High Technology, Xian, Shaanxi 710025, China

Correspondence should be addressed to Jinku Guo; gjk05@mails.tsinghua.edu.cn

Received 23 July 2014; Revised 14 September 2014; Accepted 12 October 2014

Academic Editor: Xiao-Sheng Si

Copyright ( 2015 Xiaojun Yang et al. This is an open access article distributed under the Creative Commons Attribution License, which permits unrestricted use, distribution, and reproduction in any medium, provided the original work is properly cited.

\begin{abstract}
With the advantages such as high security and far responding distance, the passive location has a broad application in military and civil domains such as radar and aerospace. However, most of the current passive location methods are based on the framework of the probability theory and cannot be used to deal with fuzzy uncertainty in the passive location systems. Though the fuzzy Kalman filter can be used in the uncertainty systems, it could not deal with the abrupt change of state like the maneuvering target which will lead to the filter divergence. Therefore, in order to track the maneuvering target in the fuzzy passive system, we proposed a robust fuzzy extended Kalman filter based on the orthogonality principle and the fuzzy filter in the paper. Conclusion can be made based on the simulation result that this new approach is more precise and more robust than the fuzzy filter.
\end{abstract}

\section{Introduction}

With the advantages such as passive receiving and far responding distance, the passive location has been broadly applied in military and civil domains, including radar and geophysics $[1,2]$. The current methods of the passive location are the unscented Kalman filter [3], the particle filter [4], and the extended Kalman filter [2]. Extended Kalman filter (EKF) [2] uses the first-order Taylor series expansion to linearize the system where measurement noise is assumed to be Gaussian. The unscented Kalman filter (UKF) [3] has better tracking precision in the nonlinear model, because it transforms the analytic integral operator into an approximate summation operator by a set of deterministic points. But both of the algorithms are not applicable to solve non-Gaussian problems. Particle filter [4] has become popular for nonlinear and/or non-Gaussian filtering and estimation. All of the above methods are based on the certainty model.

But the statistical parameters of the noise may be uncertain due to sensors drift, environmental changes, information incompleteness, and so forth. What is more, the low performance of the noise statistics estimation may lead to poor filter performance or even lead to the divergence of the filter. To solve the problem listed above, some new methods [5-12] are given.
Reference [5] proposes an optimal robust Kalman-type recursive filter for uncertain systems with autocorrelated and cross-correlated noises. Reference [7] uses the H-infinity filter to deal with the uncertain discrete-time systems. Based on the relevance vector machine and gradient descent algorithm, [8] proposes a fuzzy model identification method and [9] gives an off-online fuzzy modeling method. Reference [10] gives a new prediction model for system's behavior prediction based on belief rule base. The algorithm could use not only numerical data, but human judgmental information with uncertainty as well. Reference [6] proposes a new fuzzy extended Kalman filters (FEKF) method for mobile robots location that uses possibility distributions instead of probability distributions. In the paper, the uncertainty is not necessary to be symmetric and can be described by qualitative knowledge.

Among the above algorithms, [6] is more suitable for passive location and tracking with uncertainty that is based on quantifying uncertainty by trapezoidal possibility distributions. Being motivated by [6], [11] and [12] apply the FEKF in fault prediction and passive location in the nonlinear system with uncertainty, respectively. However, similar to the EKF, the FEKF could not estimate the state in the mismatching model system such as the maneuvering target tracking. The algorithm has poor robustness and is not 


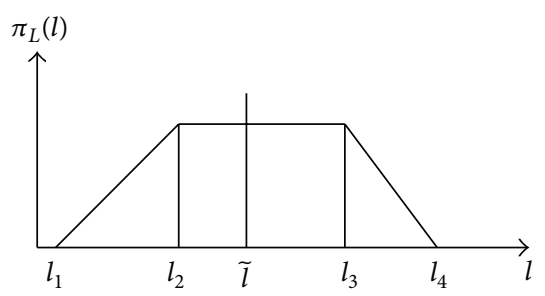

Figure 1: Possibility distribution.

suitable for maneuvering target tracking. As a result, the passive location may lose tracking ability when the target states change abruptly.

In order to overcome the above disadvantages of the FEKF, combined on the extended orthogonality principle, a robust passive location method is proposed. The algorithm can not only deal with the uncertainty, but also can be suitable for maneuvering target tracking.

We organize this paper as follows. We describe the problem in Section 2 and present the FEKF algorithm in Section 3. Combining the principle of the orthogonality, the robust fuzzy extended Kalman filter (RFEKF) is given to deal with the maneuvering target tracking in Section 4. Section 5 demonstrates the effectiveness of the RFEKF. And we summarize our conclusion in Section 6.

\section{The Problem Description}

Below is the system equation of the passive location:

$$
\begin{aligned}
& \mathbf{x}(n+1)=\mathbf{f}(\mathbf{x}(n), \mathbf{u}(n), n)+\mathbf{w}(n+1), \\
& \mathbf{z}(n+1)=\mathbf{h}(\mathbf{x}(n+1), n+1)+\mathbf{v}(n+1),
\end{aligned}
$$

where $\mathbf{x}(n+1)$ stands for the state vector covering velocity and position and so forth, $\mathbf{z}(n+1)$ is a measurement vector which can be the time or frequency of arrival, phase-difference rate of change, and so on, $\mathbf{f}(\mathbf{x}(n), n)$ is the state function, $\mathbf{h}$ is the nonlinear measurement function, $\mathbf{u}(k)$ is input vector, and $\mathbf{v}(n+1)$ and $\mathbf{w}(n+1)$ are the measurement noise and the process noise, respectively.

The noise model in most passive location algorithm is usually the probability distribution. We will further research the possibility distribution of the noise by using the trapezoidal distributions instead of Gaussian distributions in this paper. The distribution can be asymmetric which is often in the sensor. In addition, the trapezoid with two cuts can be changed to triangle with one cut, line with zero cuts, and so on. The distribution has many merits in the state estimate [6].

Here, $l$ is denoted by the fuzzy variable in the universe of discourse $L$ and $\pi_{L}(l)$ represents a trapezoidal possibility distribution [6] which is shown in Figure 1:

$$
\pi_{L}(l)= \begin{cases}1 & \forall l \in\left[l_{2}, l_{3}\right] \\ 0 & \forall l \notin\left[l_{1}, l_{4}\right]\end{cases}
$$

We define the expectation $E\{l\}$, the center of gravity $\tilde{l}$, the area of the distribution $\chi_{l}$, and the uncertainty of the distribution $U\{l\}$ as the equations below [6]:

$$
\begin{gathered}
E\{l\} \sim \Pi\left(l_{1}, l_{2}, l_{3}, l_{4}\right), \\
\tilde{l}=C\{l\}=\frac{\int l \pi_{L}(l) d l}{\chi_{l}}, \\
\chi_{l}=\int \pi_{L}(l) d l, \\
U\{l\}=C\left\{(l-\tilde{l})^{2}\right\}=\frac{\int(l-\tilde{l})^{2} \pi_{L}(l) d l}{\chi_{l}} .
\end{gathered}
$$

Remark 1. In the paper, the noise of the passive location system is under the trapezoidal distribution. Although the FEKF can solve the problems of the fuzzy uncertainty, the algorithm has poor robustness and is not suitable to deal with the maneuvering target tracking. In order to solve this problem, we will develop a robust algorithm.

\section{The Fuzzy Extended Kalman Filter}

Normally, the nonlinear system with fuzzy uncertainty can be described:

$$
\begin{aligned}
& \mathbf{x}(n+1)=\mathbf{f}(\mathbf{x}(n), \mathbf{u}(n), n)+\mathbf{w}(n+1), \\
& \mathbf{z}(n+1)=\mathbf{h}(\mathbf{x}(n+1), n+1)+\mathbf{v}(n+1),
\end{aligned}
$$

where all of the variables follow the trapezoidal distribution. Meanwhile, the variables in (1) follow the probability distribution as the Gauss distribution. $\widehat{\mathbf{x}}(n+1 \mid n)$ represents the one-step estimation value; $\widehat{\mathbf{x}}(n+1)$ and $\widehat{\mathbf{z}}(n+1)$ represent the estimation values of $\mathbf{x}(n+1)$ and $\mathbf{z}(n+1)$. What is more, $\widehat{\mathbf{x}}(n+1 \mid n)$ and $\mathbf{w}(n+1)$ are independent and $\mathbf{w}(n+1)$ and $\mathbf{v}(n+1)$ are independent too $[6,12]$. All of these parameters obey trapezoidal distribution described as follows:

$$
\begin{gathered}
E\{\mathbf{v}(n+1)\} \sim \Pi\left(\mathbf{v}_{1}(n+1), \mathbf{v}_{2}(n+1),\right. \\
\left.\mathbf{v}_{3}(n+1), \mathbf{v}_{4}(n+1)\right), \\
C(\mathbf{v}(n+1))=0, \\
U\{\mathbf{v}(n+1)\}=\mathbf{R}(n+1), \\
E\{\mathbf{w}(n+1)\} \sim \Pi\left(\mathbf{w}_{1}(n+1), \mathbf{w}_{2}(n+1),\right. \\
\left.\mathbf{w}_{3}(n+1), \mathbf{w}_{4}(n+1)\right), \\
C(\mathbf{w}(n+1))=0, \\
U\{\mathbf{w}(n+1)\}=\mathbf{Q}(n+1),
\end{gathered}
$$




$$
\begin{gathered}
E\{\widehat{\mathbf{x}}(n+1 \mid n)\} \sim \Pi\left(\widehat{\mathbf{x}}_{1}(n+1 \mid n), \widehat{\mathbf{x}}_{2}(n+1 \mid n),\right. \\
\left.\widehat{\mathbf{x}}_{3}(n+1 \mid n), \widehat{\mathbf{x}}_{4}(n+1 \mid n)\right), \\
U\{\widehat{\mathbf{x}}(n+1 \mid n)\}=\mathbf{P}(n+1 \mid n), \\
E\{\widehat{\mathbf{x}}(n)\} \sim \Pi\left(\widehat{\mathbf{x}}_{1}(n), \widehat{\mathbf{x}}_{2}(n), \widehat{\mathbf{x}}_{3}(n), \widehat{\mathbf{x}}_{4}(n)\right), \\
U\{\widehat{\mathbf{x}}(n)\}=\mathbf{P}(n), \\
E\{\widehat{\mathbf{z}}(n+1)\} \sim \Pi\left(\widehat{\mathbf{z}}_{1}(n+1), \widehat{\mathbf{z}}_{2}(n+1),\right. \\
\left.\widehat{\mathbf{z}}_{3}(n+1), \widehat{\mathbf{z}}_{4}(n+1)\right), \\
U\{\widehat{\mathbf{z}}(n+1)\}=\mathbf{S}(n+1),
\end{gathered}
$$

where $C, E$, and $U$ in the equation represent the gravity center, the expectation, and the distribution uncertainty of the fuzzy variable which are defined in (5), (4), and (7). $\mathbf{P}(n+1)$ and $\mathbf{P}(n+1 \mid n)$ are the distribution uncertainties of $\mathbf{x}(n+1)$ and $\widehat{\mathbf{x}}(n+1 \mid n)$, respectively. $\mathbf{R}(k+1), \mathbf{S}(n+1)$, and $\mathbf{Q}(n+1)$ are the distribution uncertainties of $\mathbf{w}(n+1), \widehat{\mathbf{z}}(n+1)$, and $\mathbf{v}(n+1)$, respectively.

The key steps of the algorithm are written as follows $[6,11]$.

(1) Prediction:

$$
\begin{gathered}
\widehat{\mathbf{x}}_{l}(n+1 \mid n)=\mathbf{f}\left(\widehat{\mathbf{x}}_{l}(n), n\right), \\
\widehat{\mathbf{z}}_{l}(n+1)=\mathbf{h}\left(\widehat{\mathbf{x}}_{l}(n+1 \mid k), n+1\right),
\end{gathered}
$$

where $l=1,2,3,4$.

(2) Matching measurement: the measurement $\mathbf{z}(n+1)$ can be used if

$$
\pi_{\widehat{\mathbf{Z}}}(\mathbf{z}(n+1)) \geq \varepsilon,
$$

where $\varepsilon$ is a confidence value, given by the actual conditions.

(3) Update:

$$
\begin{aligned}
& e_{l}(n+1)=\mathbf{z}_{l}(n+1)-\widehat{\mathbf{z}}_{l}(n+1), \\
& \widehat{\mathbf{x}}_{l}(n+1)=\widehat{\mathbf{x}}_{l}(n+1 \mid n)+\mathbf{K}(n+1) e_{l}(n+1), \\
& \mathbf{K}(n+1)=\mathbf{P}(n+1 \mid n) \mathbf{H}^{T}(n+1) \mathbf{S}^{-1}(n+1), \\
& \mathbf{P}(n+1)=[\mathbf{I}-\mathbf{K}(n+1) \mathbf{H}(n+1)] \mathbf{P}(n+1 \mid n),
\end{aligned}
$$

where $e_{l}(n+1)$ is estimation error,

$$
\begin{gathered}
\mathbf{P}(n+1 \mid n)=\mathbf{F}(n) \mathbf{P}(n) \mathbf{F}^{T}(n)+\mathbf{Q}(n+1), \\
\mathbf{S}(n+1)=\mathbf{H}(n+1) \mathbf{P}(n+1 \mid n) \mathbf{H}^{T}(n+1)+\mathbf{R}(n+1), \\
\mathbf{H}(n+1)=\left.\frac{\partial \mathbf{h}(\mathbf{x}(n+1), n+1)}{\partial \mathbf{x}}\right|_{\mathbf{x}(n+1)=C\{\widehat{\mathbf{x}}(n+1 \mid n)\}}, \\
\mathbf{F}(n)=\left.\frac{\partial \mathbf{f}(\mathbf{x}(n), \mathbf{u}(n), n)}{\partial \mathbf{x}}\right|_{\mathbf{x}(n)=C\{\widehat{\mathbf{x}}(n)\}}, \\
E\{e(n+1)\} \sim \Pi\left(e_{1}(n+1), e_{2}(n+1),\right. \\
\left.e_{3}(n+1), e_{4}(n+1)\right)
\end{gathered}
$$

Let us summarize the above steps as Algorithm 2.
Algorithm 2 (FEKF algorithm). Consider the following.

Step 1. Give initial values $\mathbf{P}(0)$ and $\widehat{\mathbf{x}}(0)$.

Step 2. Check (11) once we obtain the new measurement values. Calculate the new prediction values by (10), if satisfied. Otherwise, go ahead for the next new measurement.

Step 3. Use (13) to (17) to get the updated values.

Step 4. Let $n=n+1$; return to Step 2 .

Remark 3. Although the FEKF can be suitable for fuzzy system, it has poor robustness and is not suitable for maneuvering target tracking.

\section{The Fuzzy Passive Location Algorithm}

4.1. The Orthogonality Principle under the Fuzzy System. We can have the equation below by applying the orthogonality principle $[13,14]$ :

$$
E\left\{[\widehat{\mathbf{x}}(n+1)-\mathbf{x}(n+1)][\widehat{\mathbf{x}}(n+1)-\mathbf{x}(n+1)]^{T}\right\}=\min ,
$$

$$
E\left\{e(n+1+i) e^{T}(n+1)\right\}=0,
$$

where $i=1,2, \ldots$.

For the problem of fuzzy passive location given previously by (1), the above equations can be written as [11]

$$
\begin{gathered}
C\left\{[\widehat{\mathbf{x}}(n+1)-\mathbf{x}(n+1)][\widehat{\mathbf{x}}(n+1)-\mathbf{x}(n+1)]^{T}\right\}=\min , \\
\operatorname{Dep}\left\{e(n+1+i) e^{T}(n+1)\right\}=0
\end{gathered}
$$

where Deprepresents the dependency of the two variables in the fuzzy system [6].

Combining the equation below,

$$
E\{e(n)\} \sim \Pi\left(e_{1}(n), e_{2}(n), e_{3}(n), e_{4}(n)\right) .
$$

We can have the equations below:

$$
C\{e(n)\}=0, \quad n=1,2, \ldots,
$$

$$
\begin{aligned}
& \text { Dep }\left\{e(n+1+i) e^{T}(n+1)\right\} \\
&=C\{[e(n+1+i)-C\{e(n+1+i)\}] \\
&\left.\times[e(n+1)-C\{e(n+1)\}]^{T}\right\} \\
&=C\left\{e(n+1+i) e^{T}(n+1)\right\}=0 .
\end{aligned}
$$

Furthermore, we can obtain

$$
C\left\{e(n+1+i) e^{T}(n+1)\right\}=0 .
$$

From the above equations, it can be known that once there is mismatch in the model, there will be online adjustment for the gain matrix $\mathbf{K}(n+1)$ to satisfy (19). Being motivated by the principle highlighted above, we will propose a robust fuzzy passive location method to track the maneuvering target. 
4.2. A Robust Passive Location Method for the Maneuvering Target. According to the theory mentioned in Section 4.1, the maneuvering target in the fuzzy passive system can be tracked by online adjustment for $\mathbf{K}(n+1)$. As a result, (19) will be satisfied. The following equation can be obtained:

$$
\begin{aligned}
& \mathbf{P}(n+1 \mid n)=\lambda(n+1) \mathbf{F}(n) \mathbf{P}(n \mid n) \mathbf{F}^{T}(n)+\mathbf{Q}(n+1), \\
& \mathbf{K}(n+1) \\
& =\mathbf{P}(n+1 \mid n) \mathbf{H}^{T}(n+1) \\
& \quad \times\left[\mathbf{H}(n+1) \mathbf{P}(n+1 \mid n) \mathbf{H}^{T}(n+1)+\mathbf{R}(n+1)\right]^{-1},
\end{aligned}
$$

where

$$
\begin{gathered}
\lambda(n+1)=\frac{\operatorname{tr}[\mathbf{N}(n+1)]}{\operatorname{tr}[\mathbf{M}(n+1)]}, \\
\mathbf{N}(n+1)=\psi(n+1)-\mathbf{R}(n+1) \\
-\mathbf{H}(n+1) \mathbf{Q}(n+1) \mathbf{H}^{T}(n+1), \\
\mathbf{M}(n+1)=\mathbf{H}(n+1) \mathbf{F}(n) \mathbf{P}(n) \mathbf{F}^{T}(n) \mathbf{H}^{T}(n+1), \\
\psi(n+1) \quad n=0 \\
= \begin{cases}C\left\{e(1) e^{T}(1)\right\}, \\
\frac{\omega \psi(n)+C\left\{e(n+1) e^{T}(n+1)\right\}}{1+\omega}, & n \geq 1,\end{cases}
\end{gathered}
$$

where $\lambda(n+1)$ and tr denote the fading factor and the matrix trace, respectively, and $\omega$ is the forgetting factor which should satisfy $0 \leq \omega \leq 1$. Generally, $\omega=0.95[10,13]$.

We can summarize the above procedures into Algorithm 4 named the robust fuzzy extended Kalman filter (RFEKF).

Algorithm 4 (RFEKF algorithm). Consider the following.

Step 1. Give initial values $\mathbf{P}(0)$ and $\widehat{\mathbf{x}}(0)$.

Step 2. Check (11) once we obtain the new measurement values. Calculate the new prediction values by (10), if satisfied. Otherwise, go ahead for the next new measurement.

Step 3. Obtain the gain matrix by using (25)-(26).

Step 4. Obtain the updated value by using (13)-(14).

Step 5. Let $n=n+1$; return to Step 2 .

In the RFEKF, gain matrix $\mathbf{K}(n+1)$ is calibrated appropriately by $\lambda(n+1)$ when the stable states change abruptly. That is, the tracking performance of RFEKF is more robust and suitable for the maneuvering target tracking than the FEKF.

Remark 5. In the RFEKF, introducing the orthogonality principle, the gain matrix $\mathbf{K}(n+1)$ could be adjusted online, if the model has mismatch. As a result, the RFEKF could not only be applied for the fuzzy uncertainty, but also has stronger robustness than that of the FEKF. In a word, the RFEKF can deal with the maneuvering target tracking of the fuzzy passive location system.

Moreover, with the above RFEKF, we can obtain a robust fuzzy passive location algorithm for maneuvering target in the passive location system given by (1) and (2). The robust algorithm is armed with the advantages of the RFEKF and is more robust. Thus, the algorithm is applicable for maneuvering target tracking. In the following simulation studies, we will compare the RFEKF with the FEKF in the fuzzy passive location systems to demonstrate the effectiveness of this algorithm.

Based on [11], the criterions are given.

Criterion 1. The true values should be in the zone of the trapezoidal distribution zone. Moreover, the smaller the trapezoidal zone is, the more effective the algorithm is.

Criterion 2. The algorithm is more effective if the error is smaller.

\section{Simulation}

In order to demonstrate the effectiveness of the proposed algorithm, we give a passive location model. The measurements in the model are composed of frequency rate of change $\dot{f}_{d}$, phase-difference rate of change $\dot{\varphi}$, and phase-difference $\varphi$ [15]. In the simulation, the observer is located at the origin and the target keeps moving in a constant speed.

We can use the equations below as the passive location model:

$$
\mathbf{x}(n+1)=\left[\begin{array}{cc}
\mathbf{I}_{2} & \mathbf{I}_{2} T_{s} \\
0 & \mathbf{I}_{2}
\end{array}\right] \mathbf{x}(n)+\mathbf{w}(n+1),
$$

$$
\mathbf{z}(n+1)=\left[\begin{array}{c}
\varphi(n+1) \\
\dot{\varphi}(n+1) \\
\dot{f}_{d}(n+1)
\end{array}\right]+\mathbf{v}(n+1)
$$

where $\mathbf{I}_{2}$ is the identity matrix of second order, $\mathbf{x}=[x, \dot{x}, y, \dot{y}]$ is the target state, $x, \dot{x}, y$, and $\dot{y}$ represent horizontal position, horizontal velocity, vertical position, and vertical velocity, $f_{0}$, respectively, denote the frequency, $k_{0}$ is the phase-difference coefficient, and

$$
\begin{gathered}
\varphi(n)=k_{0} f_{0} \frac{x(n)}{\sqrt{x^{2}(n)+y^{2}(n)}}, \\
\dot{\varphi}(n)=-k_{0} f_{0} y(n) \frac{-\dot{x}(n) y(n)+\dot{y}(n) x(n)}{\left[x^{2}(n)+y^{2}(n)\right]^{3 / 2}},
\end{gathered}
$$




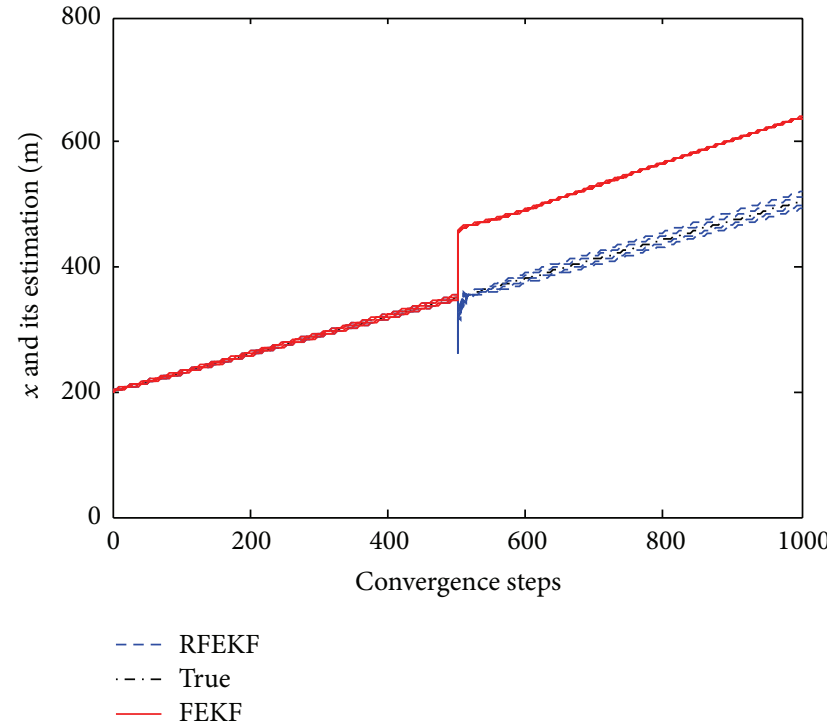

(a)

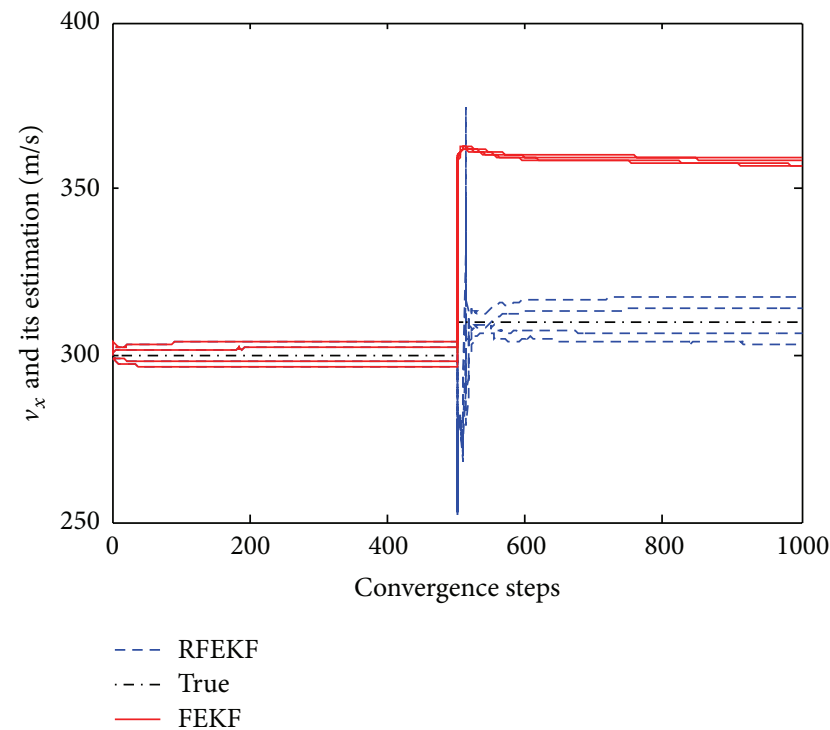

(c)

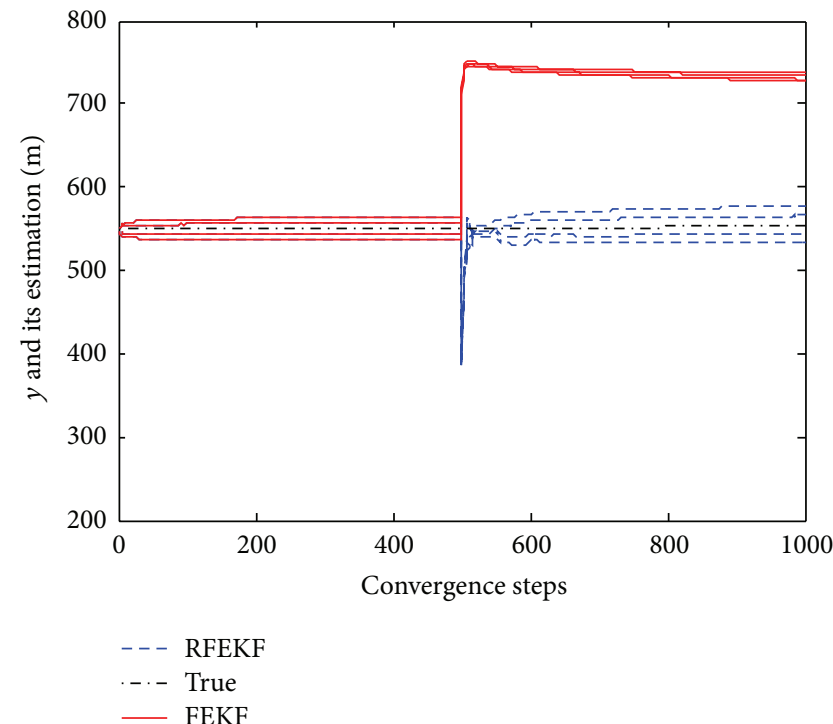

(b)

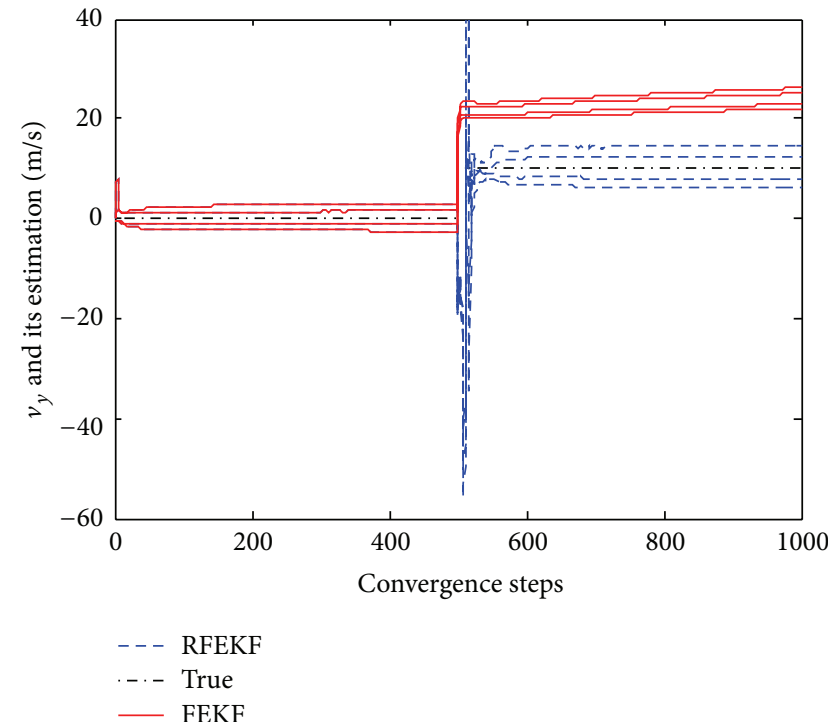

(d)

FIgURE 2: Using the 100-time Monte-Carlo simulation method to compare the estimation value of the target applying the FEKF and the RFEKF. (a) Estimation values of the horizontal position, (b) estimation values of the horizontal velocity, (c) estimation values of the vertical position, (d) estimation values of the vertical velocity. The actual values of the target are represented by the dotted and dashed line; the value of the FEKF is represented by the red solid line and that of the RFEKF is represented by the blue dashed line.

$$
\begin{gathered}
\dot{f}_{d}(n)=-\frac{[-\dot{x}(n) y(n)+\dot{y}(n) x(n)]^{2}}{\lambda\left[x^{2}(n)+y^{2}(n)\right]^{3 / 2},} \\
E\{\mathbf{w}\} \sim \Pi(-0.004,-0.002,0.002,0.004), \\
E\{\mathbf{v}\} \sim \Pi(-0.001,-0.0005,0.0005,0.001) .
\end{gathered}
$$

In this simulation, the initial state is $\dot{x}=300 \mathrm{~m} / \mathrm{s}$ and $\dot{y}=0 \mathrm{~m} / \mathrm{s}$. When $t=501$, the target states change abruptly. We suppose that $\dot{x}(501)=310 \mathrm{~m} / \mathrm{s}$ and $\dot{y}(501)=10 \mathrm{~m} / \mathrm{s}$. We use the 100-time Monte-Carlo simulation for the experiments below. Figure 2 exhibits the estimation of the state $\mathbf{x}$ between the FEKF and RFEKF. In Figure 2, you can see the actual values in the dotted and dashed line. The estimation value of the RFEKF is the blue dashed line and the value of the FEKF is the red solid line. Figures 3 and 4 exhibit the estimation error of the state between the RFEKF and the FEKF, respectively.

In Figure 2, when $t \in\left[\begin{array}{ll}0 & 500\end{array}\right]$, the true state values of the target are all in the trapezoidal distribution zones of the FEKF and RFEKF. By Criterion 1, it is shown that both of the algorithms can track the nonmaneuvering target. But after the states of the target change abruptly $(t=501)$, the actual state values are out of the trapezoidal zones of the FEKF but in 


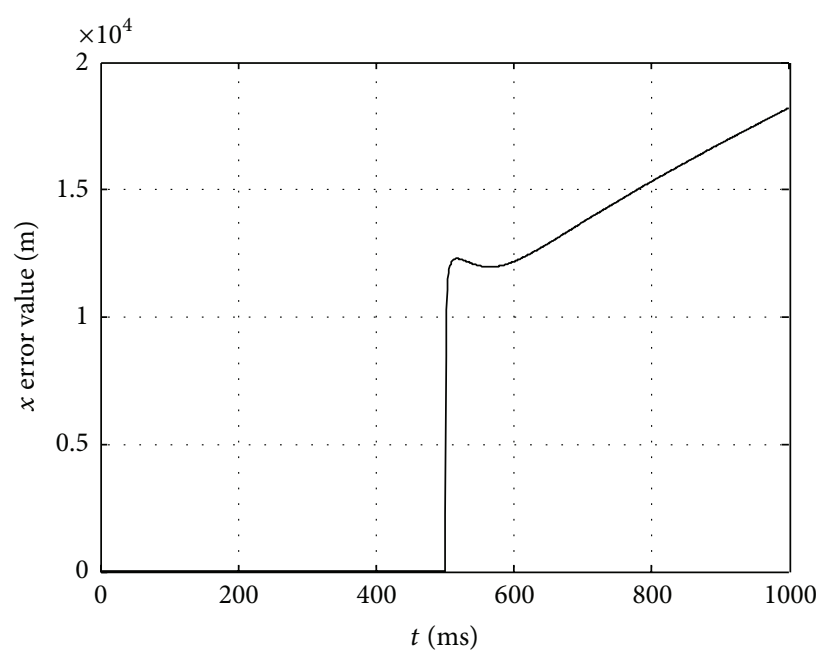

(a)

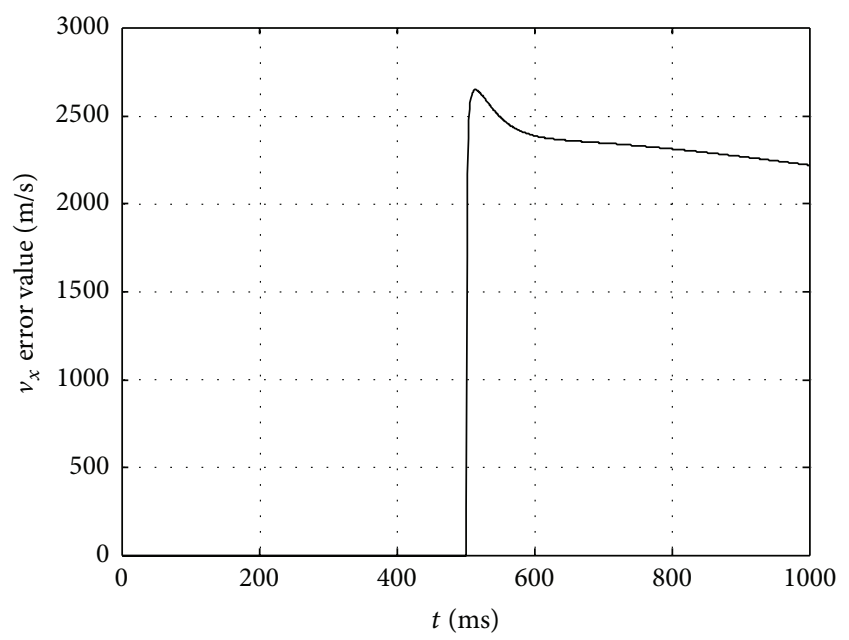

(c)

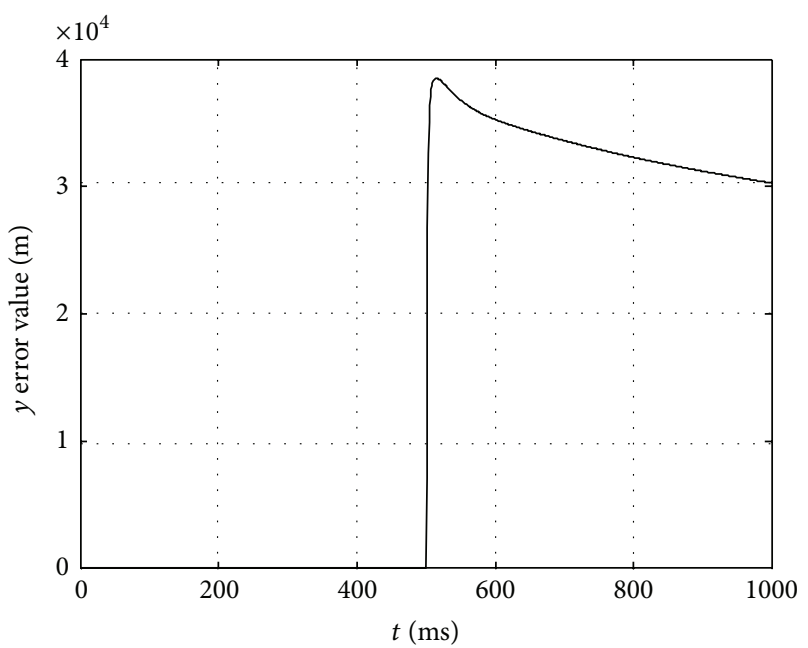

(b)

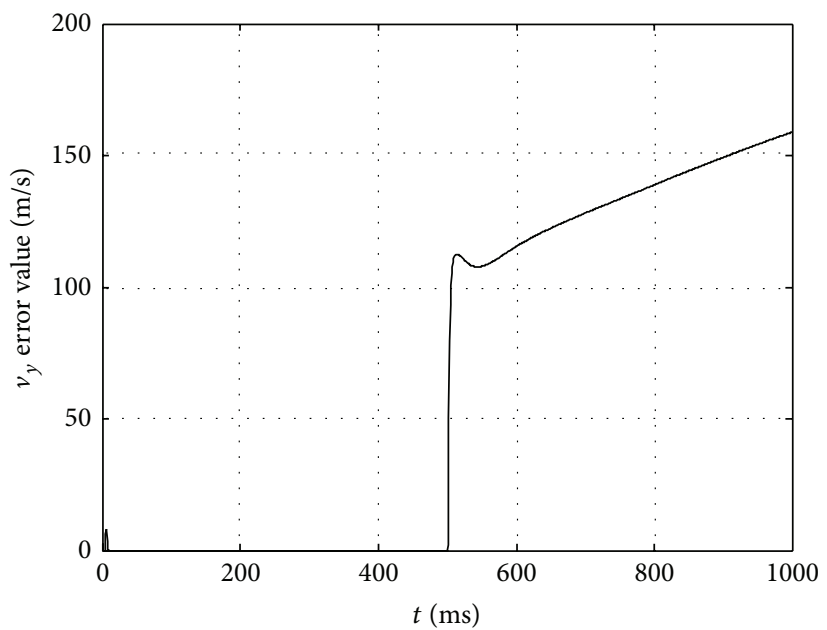

(d)

FIGURE 3: Using the 100-time Monte-Carlo simulation method to get the error of the FEKF. (a) Error of the horizontal position, (b) error of the horizontal velocity, (c) error of the vertical position, and (d) error of the vertical velocity.

that of the RFEKF. Thus, by Criterion 1, it is shown that the RFEKF is suitable for the maneuvering target tracking, while the FEKF is not.

In Figure 3, the FEKF is convergent when $t \in\left[\begin{array}{ll}0 & 500\end{array}\right]$, while the algorithm is divergent after the states of the target change abruptly $(t=501)$. In Figure 4 , the RFEKF is convergent when $t \in\left[\begin{array}{ll}0 & 500\end{array}\right]$, and the error values are huge in the beginning after the states of the target change abruptly $(t=501)$. However, it will be back to convergent after some steps. Compare Figure 3 with Figure 4; we can draw the conclusion that when the target is nonmaneuvering, both of the algorithms are convergent, and when the target is maneuvering, the FEKF is divergent and the RFEKF is convergent after some steps. By Criterion 2, it is shown that the RFEKF is more effective and suitable for the maneuvering target tracking.

In a word, simulations show that the RFEKF has stronger robustness and is more suitable to track the maneuvering target.

\section{Conclusions}

In order to track the maneuvering target in the uncertain passive location system, the robust passive location algorithm is proposed based on the orthogonality principle and the fuzzy extended Kalman filter. It is different from other passive location methods as the noise is described by the trapezoidal possibility distributions in this paper instead of the Gaussian probability distributions. In the algorithm, the uncertainty can be built by qualitative knowledge and is not necessary to be symmetric.

The simulation results demonstrate that both of the FEKF and the RFEKF can track the nonmaneuvering target. But the RFEKF can track the maneuvering target while the FEKF cannot. In a word, the RFEKF could not only deal with the fuzzy passive system, but also have stronger robustness and is more suitable for the maneuvering target tracking than the FEKF. 


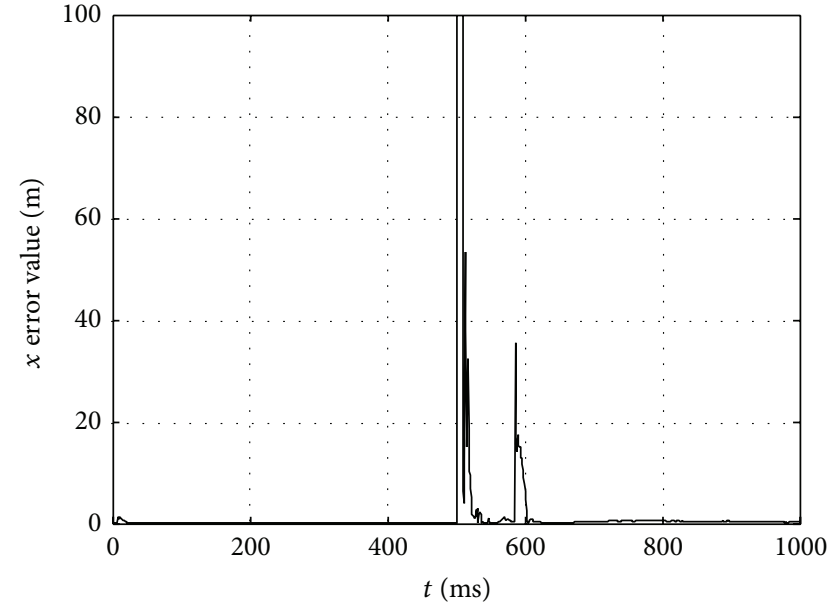

(a)

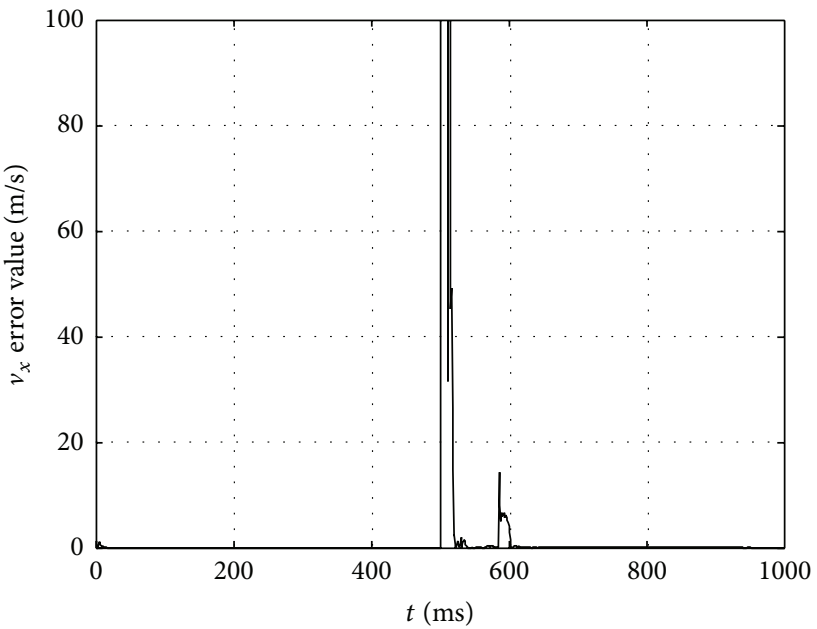

(c)

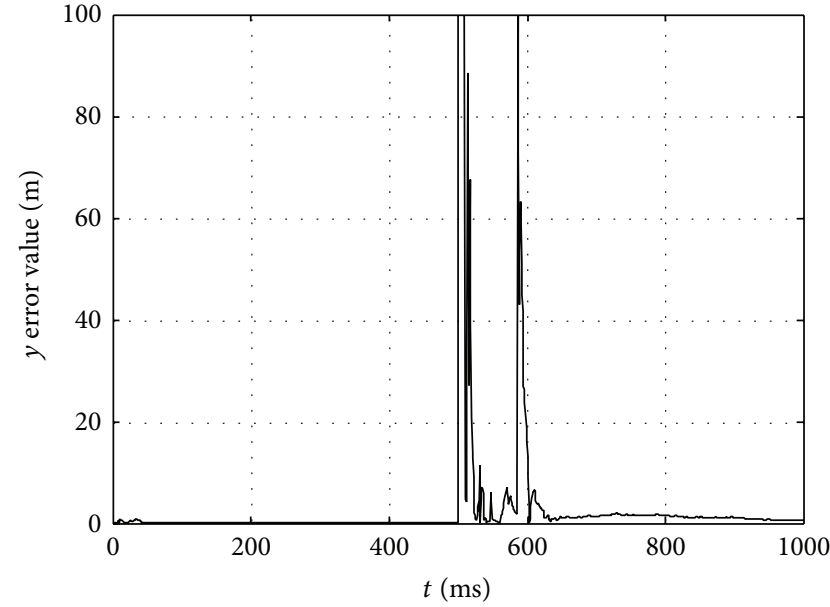

(b)

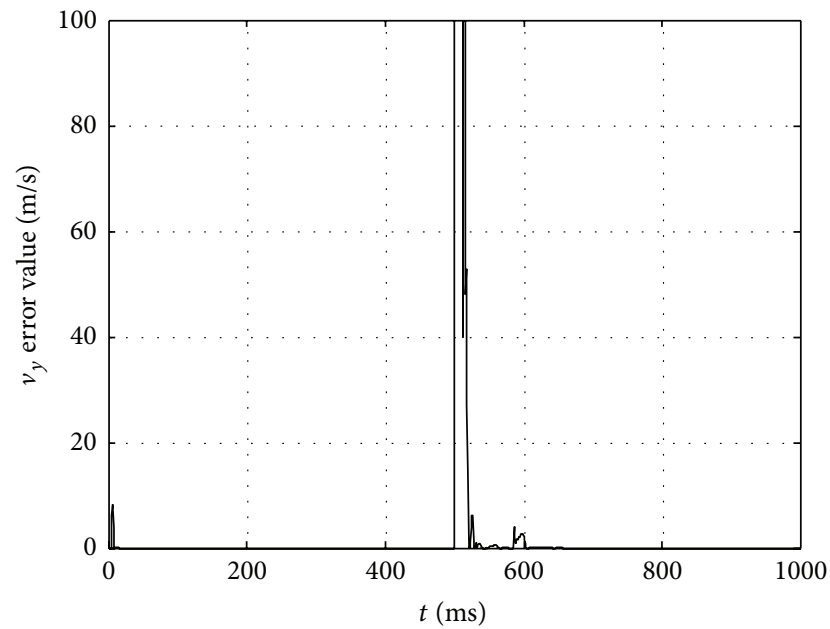

(d)

Figure 4: Using the 100-time Monte-Carlo simulation method to get the error of the RFEKF. (a) Error of the horizontal position, (b) error of the horizontal velocity, (c) error of the vertical position, and (d) error of the vertical velocity. The error values at the abrupt change time are huge. In order to see the effect of the convergence, the vertical limit of this figure was set as 100 artificially.

Fuzzy extended Kalman filter in different environments such as high nonlinear, data loss will be topics of further study. Meanwhile, it will be meaningful to further study the convergence property of the fuzzy filter.

\section{Conflict of Interests}

The authors declare that there is no conflict of interests regarding the publication of this paper.

\section{Acknowledgments}

This research was financially supported by the National Science Foundation (61304240, 61202332, and 61201120) and China Postdoctoral Science Foundation (2014M552589, 2012M521904, and 2012M521905).

\section{References}

[1] J. Shen, F. Molisch, and J. Salmi, "Accurate passive location estimation using TOA measurements," IEEE Transactions on Wireless Communications, vol. 11, no. 6, pp. 2182-2192, 2012.

[2] X. F. Meng, "Analysis of the effects of passive location based on the extended Kalman filtering algorithm," Electronic Science and Technology, vol. 25, no. 3, pp. 25-27, 2012.

[3] S. K. Rao, "Doppler-bearing passive target tracking using a parameterized unscented kalman filter," IETE Journal of Research, vol. 56, no. 1, pp. 69-75, 2010.

[4] H.-W. Li and J. Wang, "Particle filter for manoeuvring target tracking via passive radar measurements with glint noise," IET Radar, Sonar and Navigation, vol. 6, no. 3, pp. 180-189, 2012.

[5] J. Feng, Z. Wang, and M. Zeng, "Distributed weighted robust Kalman filter fusion for uncertain systems with autocorrelated and cross-correlated noises," Information Fusion, vol. 14, no. 1, pp. 78-86, 2013. 
[6] F. Matía, A. Jiménez, B. M. Al-Hadithi, D. Rodríguez-Losada, and R. Galán, "The fuzzy Kalman filter: state estimation using possibilistic techniques," Fuzzy Sets and Systems, vol. 157, no. 16, pp. 2145-2170, 2006.

[7] K.-Z. Han and X.-H. Chang, "Parameter-dependent robust $H_{\infty}$ filter design for uncertain discrete-time systems with quantized measurements," International Journal of Control, Automation and Systems, vol. 11, no. 1, pp. 194-199, 2013.

[8] C. H. Hu, Z. Q. Wang, Z. J. Zhou, and X. S. Si, "An RVM fuzzy model identification method and its application to fault prediction," Acta Automatica Sinica, vol. 37, no. 4, pp. 503-512, 2011.

[9] Z. Wang, C. Hu, W. Wang, X. Si, and Z. Zhou, "An offonline fuzzy modelling method for fault prognosis with an application," in Proceedings of the 3rd Annual IEEE Prognostics and System Health Management Conference (PHM '12), pp. 1-7, Beijing, China, May 2012.

[10] X.-S. Si, C.-H. Hu, J.-B. Yang, and Z.-J. Zhou, "A new prediction model based on belief rule base for systems's behavior prediction," IEEE Transactions on Fuzzy Systems, vol. 19, no. 4, pp. 636651, 2011.

[11] Z. J. Zhou, C. H. Hu, H. D. Fan, and J. Li, "Fault prediction of the nonlinear systems with uncertainty," Simulation Modelling Practice and Theory, vol. 16, no. 6, pp. 690-703, 2008.

[12] X. Yang, H. Zou, F. Lu, J.-J. Ding, Z. Zhou, and D. Liu, "Passive location of the nonlinear systems with fuzzy uncertainty," Simulation Modelling Practice and Theory, vol. 18, no. 3, pp. 304316, 2010.

[13] X. Li, Z. Xu, and D. Zhou, "Chaotic secure communication based on strong tracking filtering," Physics Letters A, vol. 372, no. 44, pp. 6627-6632, 2008.

[14] X. Wang, X. He, Z. Wang, and D. Zhou, "Sensor fault estimation for a class of nonlinear networked systems," in Proceedings of the 25th Chinese Control and Decision Conference (CCDC '13), pp. 4967-4972, Hefei, China, May 2013.

[15] X. J. Yang, G. Liu, and J. Guo, "A single observation passive location algorithm based on phase-difference and doppler frequency rate of change," in Proceedings of the IEEE International Conference on Systems, Man and Cybernetics (SMC '08), pp. 1309-1313, Singapore, October 2008. 


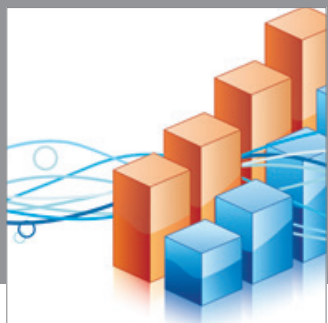

Advances in

Operations Research

mansans

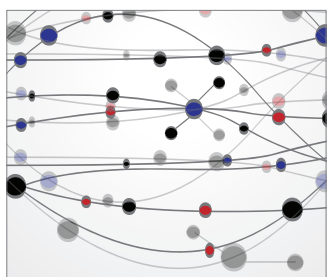

The Scientific World Journal
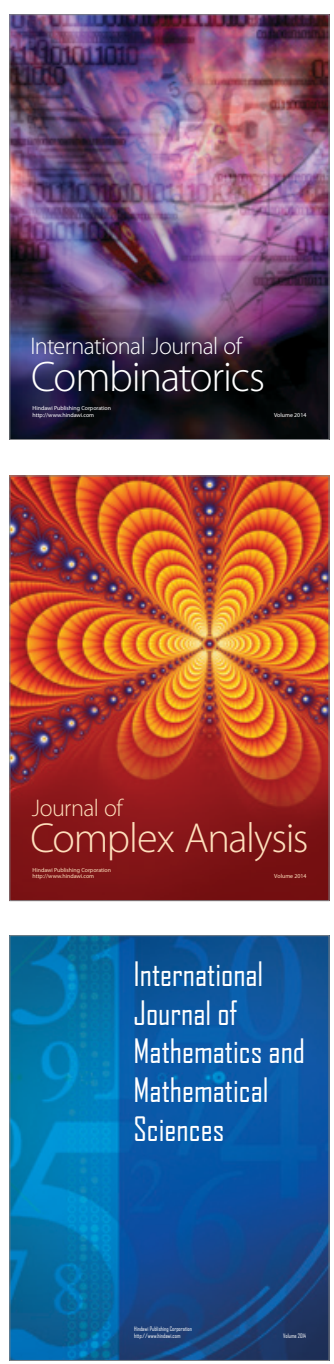
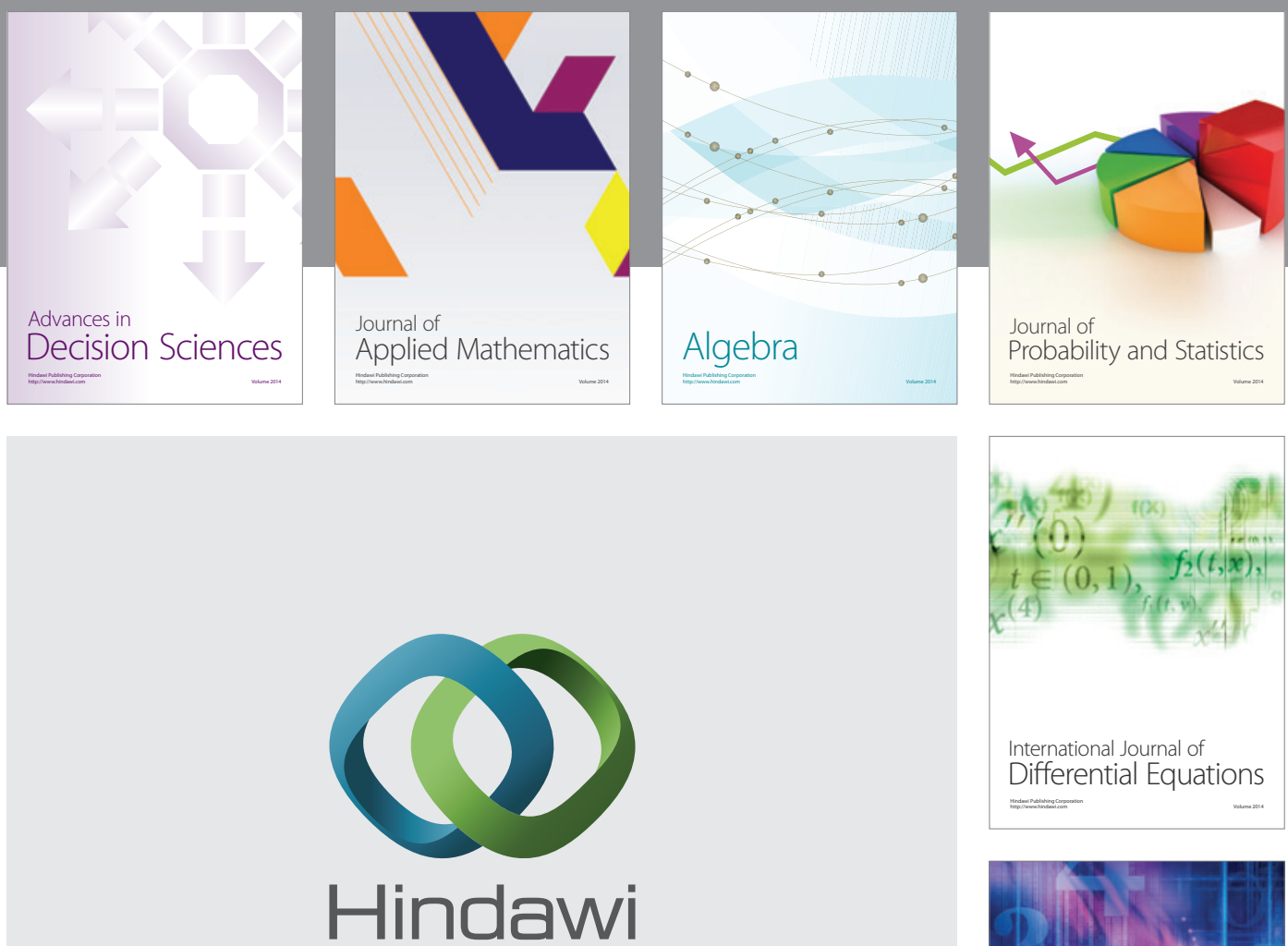

Submit your manuscripts at http://www.hindawi.com
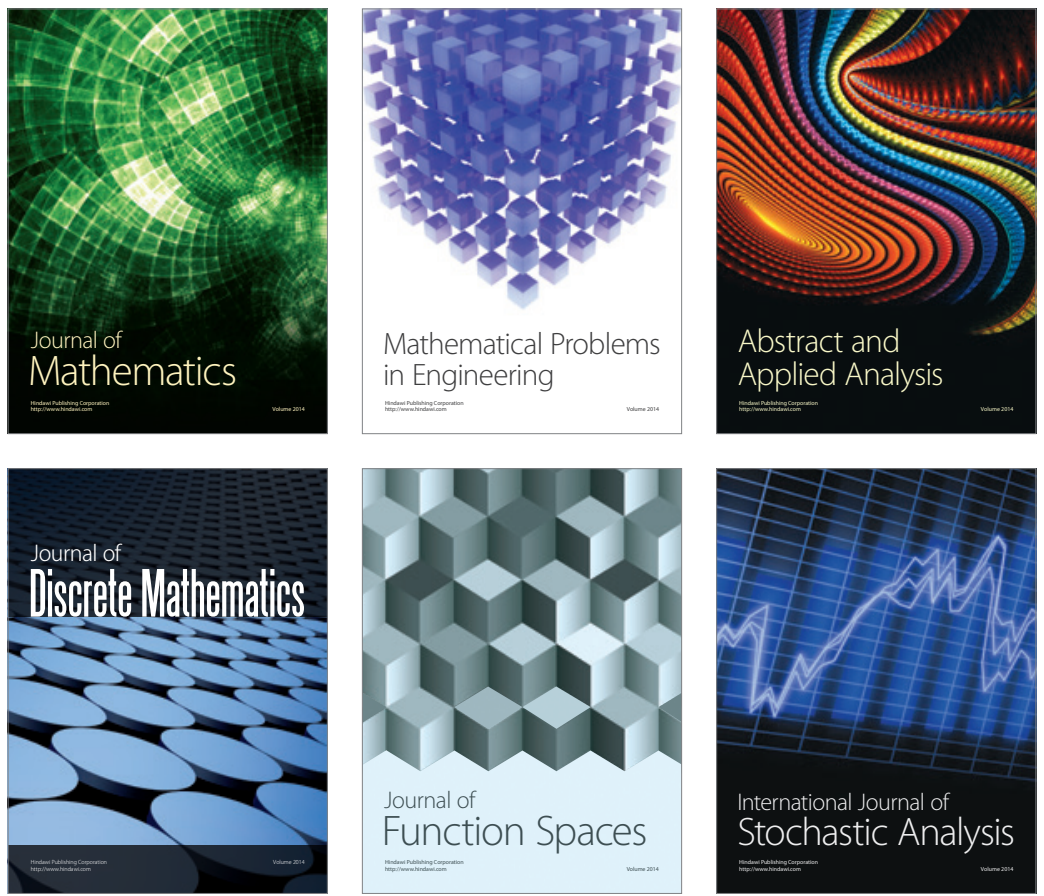

Journal of

Function Spaces

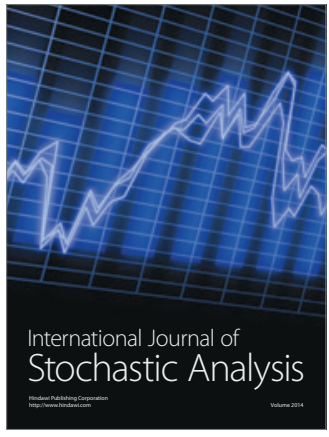

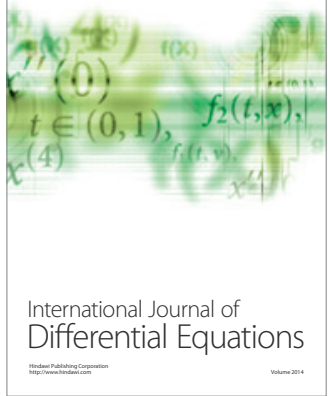
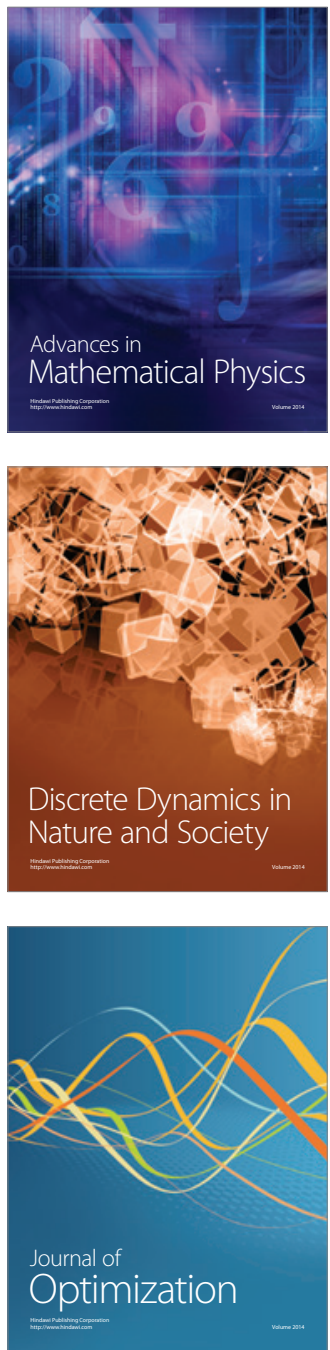